\title{
Design of subwavelength optical fibre for low-loss Terahertz transmission
}

N. F. Ren

M. Y. Chen

miniyoung@163.com

B. Sun

L. Ge
School of Mechanical Engineering, Jiangsu University, Zhenjiang 212013, Jiangsu Province, China

School of Mechanical Engineering, Jiangsu University, Zhenjiang 212013, Jiangsu Province, China

School of Mechanical Engineering, Jiangsu University, Zhenjiang 212013, Jiangsu Province, China

School of Mechanical Engineering, Jiangsu University, Zhenjiang 212013, Jiangsu Province, China

A low-loss Terahertz $(\mathrm{THz})$ transmission optical fibre with a subwavelength core is reported in this article. The main fibre is composed of a subwavelength solid polymer core and a tube. The tube is used to prevent the extending of THz wave to the external environment. Two solid ends are introduced to suspend the subwavelength core in the air. The solid fibre ends are found have low splicing losses with the main fibre. The proposed fibre provides a simple technique for the transmission of THz wave in a short distance.

[DOI: http://dx.doi.org/10.2971/jeos.2013.13068]

Keywords: THz transmission, optical fibre, absorption loss

\section{INTRODUCTION}

$\mathrm{THz}$ radiation band, which is defined as the electromagnetic wave with frequency ranging from $0.1 \mathrm{THz}$ up to $10 \mathrm{THz}$ ) $[1,2]$, has brought potential applications in many fields, such as spectroscopy, biological imaging, environmental monitoring, and military security [3]-[7], etc. Another new area is the application of the terahertz band in the future communication systems [2]. Unfortunately, $\mathrm{THz}$ radiation is strongly absorbed by most kind of materials, which gives a challenge to achieve low-loss $\mathrm{THz}$ transmission waveguide. Polytetrafluoroethylene (PTFE) is one of the most commonly used lowloss waveguide material, but its absorption loss is $130 \mathrm{~dB} / \mathrm{m}$, which is still very large for most of applications [8]. As a result, the lack of waveguide materials well suited for guided propagation at $\mathrm{THz}$ frequencies limits the practical application of $\mathrm{THz}$ technology. Fortunately, the propagation of THz wave in dry air has low loss, which means low-loss $\mathrm{THz}$ waveguiding can be realized by the design of novel optical fibre which introduces large fractional power in air [9]. For example, air-core photonic bandgap fibres have the potential to guiding $\mathrm{THz}$ wave in the air cores, which can be an nice choice for $\mathrm{THz}$ guiding [10, 11]. Honeycomb bandgap THz fiber composed of porous-core shows the possibility of achieving a propagation loss of $20 \mathrm{~dB} / \mathrm{m}$ at $1 \mathrm{THz}$ [10]. Hollow-core $\mathrm{THz}$ Bragg fibers were shown to have straight waveguide propagation losses lower than $0.05 \mathrm{~cm}^{-1}$ at certain peak transmission frequenceies [11]. Recently, porous polymer fibres designed for low-loss $\mathrm{THz}$ guiding have been proposed and investigated $[8,12,13]$. Porous dielectric subwavelength $\mathrm{THz}$ fibers were demonstrated to guide with losses as low as $0.02 \mathrm{~cm}^{-1}$, also at low frequency of $0.14-0.21 \mathrm{THz}$ and in the $0.06-0.14 \mathrm{THz}$ [13]. The suspended small solid core fiber offers very low-loss $\left(0.02 \mathrm{~cm}^{-1}\right)$ single mode guiding, although at a low frequency range of $0.28-0.48 \mathrm{THz}$, where the absorption loss of the bulk material is lower than $0.2 \mathrm{~cm}^{-1}$ [14]. By using polymer tube, it's also possible to guide $\mathrm{THz}$ wave with low transmission loss $[9,15]$. However, the external environment has a direct effect on the $\mathrm{THz}$ transmission based on porous fibres or subwavelength tube, owing to the fact that $\mathrm{THz}$ wave is extending to the air outside the fibres. In this paper, we provide a simple technique to realize a low-loss $\mathrm{THz}$ fibre. The low-loss transmission is based on total internal-reflection theory, and all the energy is guided in the proposed fibre. Therefore, the influence of external environment can be totally avoided.

\section{NUMERICAL SIMULATION}

The configuration of the proposed compact $\mathrm{THz}$ optical fibre is shown in Figure 1(a). The main fibre is composed of a subwavelength core which is surrounded by the air cladding and the outer solid cladding. The fibre core is suspended in air by the solid jackets at two ends. The solid outer cladding is used to isolate the guided mode from the outer environment. The fabrication process can be listed as follows. Firstly, the subwavelength core, the outer cladding, and the supporting jackets should be prepared. Secondly the subwavelength core will be inserted into the outer cladding, and the two cores will perforate the two jackets and be pushed straight. The jackets will be sealed to form the solid ends and the overlength core will be cut down. Apparently, the solid ends should have short lengths to keep the transmission loss of the whole fibre at low level. A elementary experiment shows that a short length of 


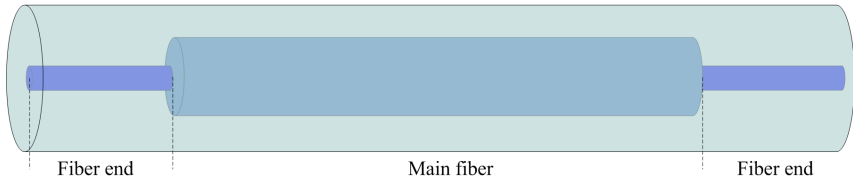

(a)

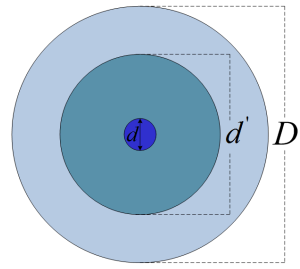

(b)

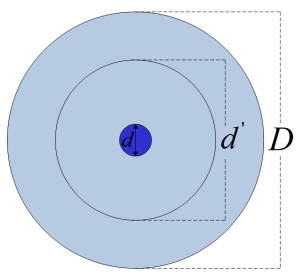

(c)
FIG. 1 (a) Configuration of the proposed THz fibre, (b) the cross-section of the main fibre, which is composed of a solid core, air hole cladding, and the outer solid cladding, (c) the solid fibre end, which is composed of a solid core, and a solid cladding.

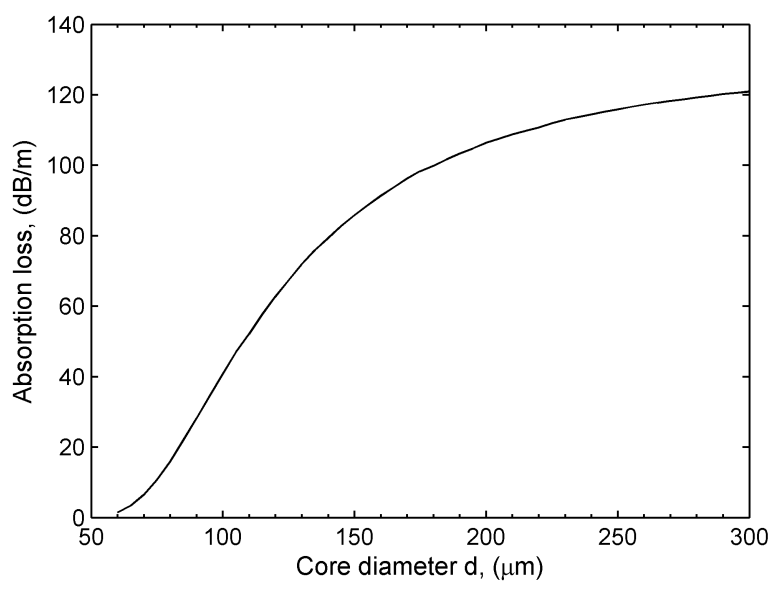

FIG. 2 The absorption loss of the subwavelength fibre.

5 10 $\mathrm{mm}$ for the ends should be long enough to suspend the subwavelength core. Here the material of subwavelength core is set to be PTFE, which has a low absorption loss of $130 \mathrm{~dB} / \mathrm{m}$ and refractive index of 1.5 at the wavelength of $300 \mu \mathrm{m}$. The refractive index of the cladding is set as 1.48 . We solve the modes of the proposed fibres by a full vectorial finite-element method with anisotropic perfect matched layer boundary conditions [16]. Firstly, we would determine the appropriate size of the subwavelength core. The modal absorption loss $L$ can be defined as $L=\epsilon \times \eta$, where $\epsilon$ is the material loss and $\eta$ is the power fractions in the solid material. It is evident that more power fraction will be confined in air with the reduction of core diameter $d$. Figure 2 shows the absorption loss of a subwavelength fibre without the solid cladding. It is found that the modal absorption loss is approaching that of the material loss when the diameter of the core is $300 \mu \mathrm{m}$, which shows the strong confinement ability of the surrounding air. The modal absorption loss is $6.4 \mathrm{~dB} / \mathrm{m}$ when $d$ is decreased to $70 \mu \mathrm{m}$. On the other hand, when the core diameter reduces, the mode field diameter increases. The result is shown in Figure 3. We can see the $\mathrm{THz}$ wave propagating in a subwavelength core optical fibre would be very sensitive to the variation of the environment, which limits its application.

We introduce a solid cladding to isolate the $\mathrm{THz}$ wave from the outer environment. Figure 4 shows the modal absorption

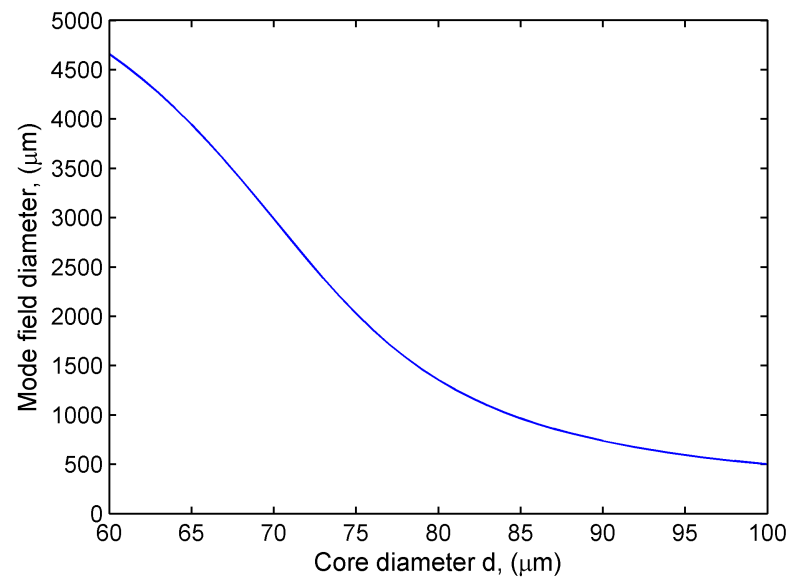

FIG. 3 The mode field diameter of the subwavelength optical fibre as a function core diameter $d$.

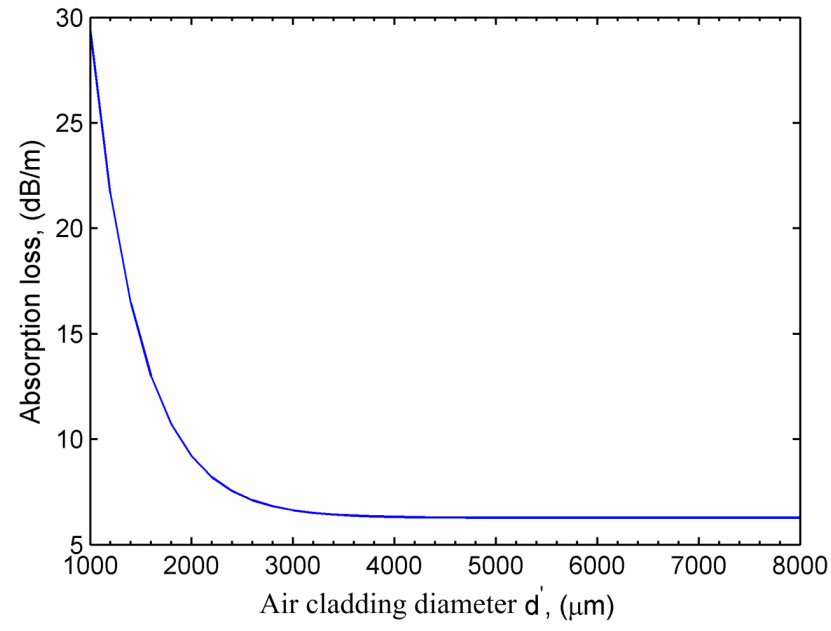

FIG. 4 The modal absorption loss as a function of the diameter of air cladding $d^{\prime}$.

loss as a function of the diameter of air cladding $d^{\prime}$. The outer diameter of the solid cladding $D$ is set as $7 \mathrm{~mm}$. It can be seen from Figure 4 that the diameter of air cladding has strong influence on the absorption loss at a small size. When the diameter is large enough, the further increase of the diameter has little influence on the absorption loss. In particular, the absorption loss can be lower than $6.5 \mathrm{~dB} / \mathrm{m}$ when the air cladding diameter is larger than $5 \mathrm{~mm}$. The transmission characteristics of the main fibre and two fibre ends have been investigated through a full-vectorial beam propagation method (FV-BPM) [17] with transparent boundary conditions [18]. Owing to the fact that little energy is propagating at the outer cladding, the outer diameter of the solid cladding has little influence on the absorption loss of the $\mathrm{THz}$ wave. Based on the above investigation, the corresponding parameters are chosen as follows. The core diameter $d$ is $70 \mu \mathrm{m}$, the external diameter $d^{\prime}$ is $5 \mathrm{~mm}$ and the value of $D$ is $7 \mathrm{~mm}$. To suspend the subwavelength core in the air cladding, the air cladding at the two ends of the fibre will be replaced with the solid supporting cladding, the configuration is shown in Figure 1. Since the two ends use the same core as the main fibre, the main difference between the ends and the main fibre comes from the cladding. For the input port, owing to the fact that $\mathrm{THz}$ wave propagates from the low NA(numerical aperture) waveguide to the high NA waveguide, low splicing loss is expected. Ow- 


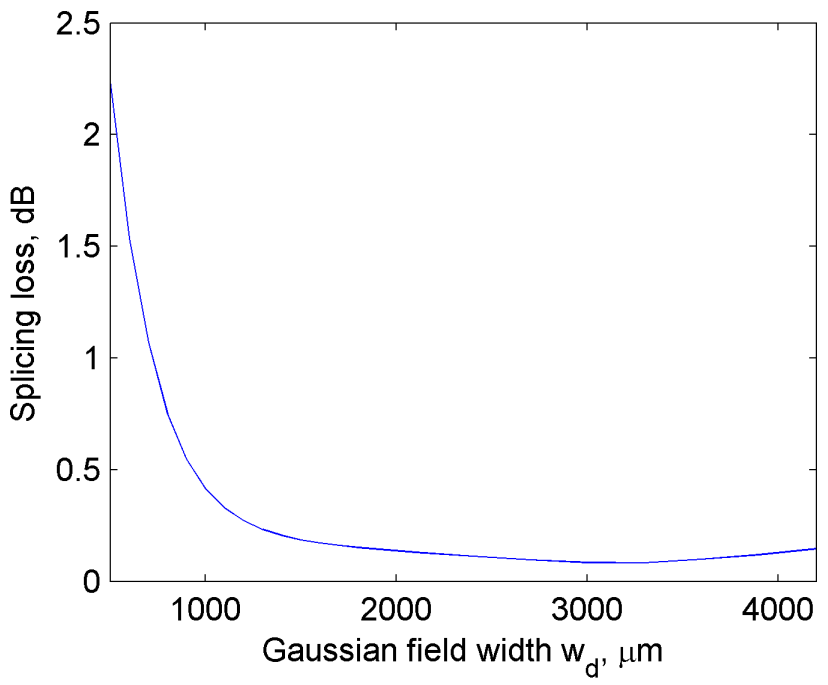

FIG. 5 Splicing loss between the solid input end and the main fibre as a function of THz source width.

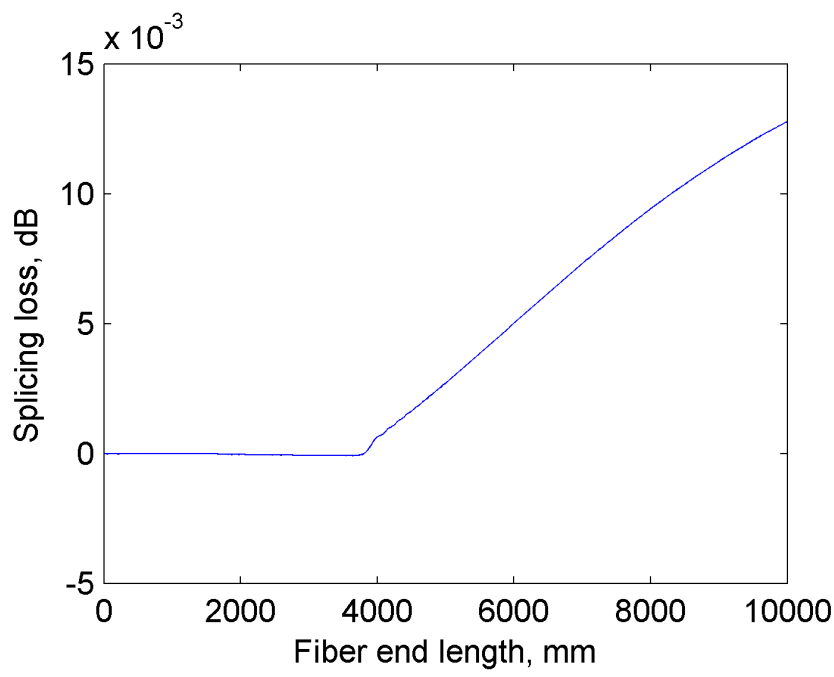

FIC. 7 Splicing loss between the the main fibre and the output end as a function of THz source width.

ing to the fact that the solid end is relative short, the input field reaches the main fibre before it become stable. Therefore, the splicing loss is depended on the source field. Here we assume a Gaussian type source. The splicing loss as a function of the Guassian field width $w_{d}$ is plotted in Figure 5. It's found the Gaussian field width influence the splicing loss. To investigate that, we plotted in Figure 6 the mode field diameter of the $\mathrm{THz}$ wave at the interface between the solid input end and the main fibre. It should be noted that the mode field diameter of the main fibre is $2987 \mu \mathrm{m}$. We can see when the mode field at the interface matches the mode field of the main fibre, the splicing loss is low. At a low source width, the mode field dissipates very fast. For example, the mode field expands from the original input field width of $500 \mu \mathrm{m}$ to $2570 \mu \mathrm{m}$ at the interface between the solid input end and the main fibre. Which is the reason of large splicing loss at low source field width.

At the output port, the splicing loss can still be very low. As shown in Figure 7, low splicing loss can be achieved for a wide range of solid fibre length. Certainly, if the solid end is very long (generally $10 \mathrm{~mm}$ or longer), the $\mathrm{THz}$ wave will be

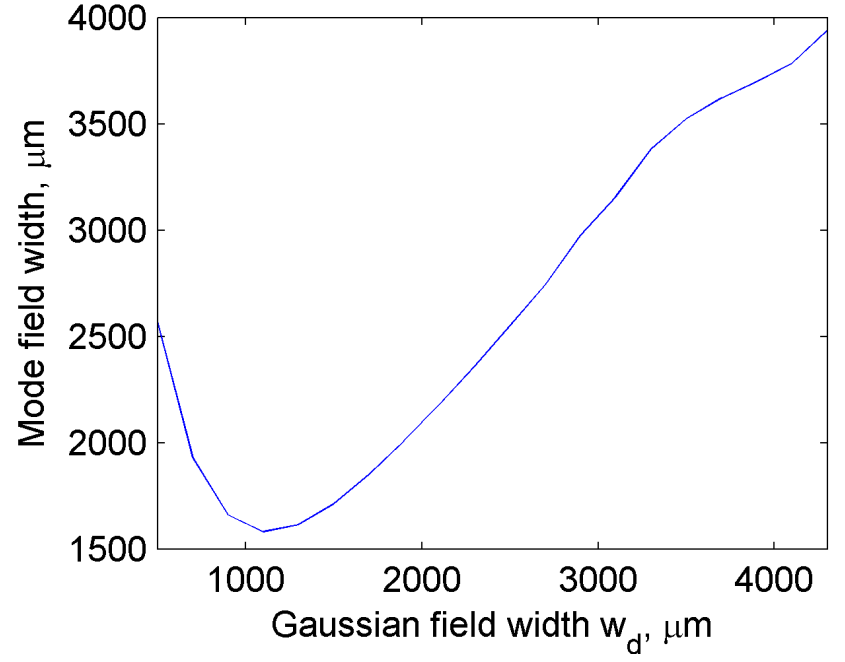

FIG. 6 Mode field diameter at the interface between the solid input end and the main fibre.

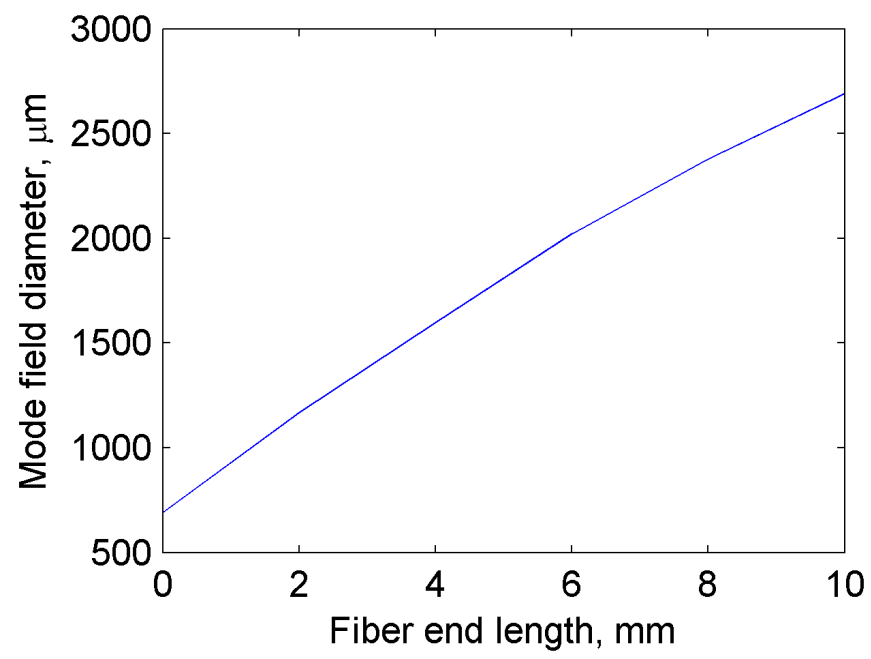

FIG. 8 Mode field diameter at the interface between the main fibre and the solid output end.

extended to the entire fibre eventually, and taking the outer air as cladding. Therefore, the output end should be short enough. The mode field diameter in the solid end will expand as shown in Figure 8. This means we can choose the appropriate solid fibre length to achieve the desired mode field at the output port.

Although the solid fibre ends have high absorption loss, which will increase the transmission loss of the fibre, it's still possible to achieve low loss transmission. For exmaple, assume a $\mathrm{THz}$ fibre with a length of $0.5 \mathrm{~m}$ and $5 \mathrm{~mm}$ length of each fibre end. The absorption loss of the main fibre is $4.6 \mathrm{~dB} / \mathrm{m}$ for the fibre with $d=70 \mu \mathrm{m}, d^{\prime}=5 \mathrm{~mm}$ and $D=7 \mathrm{~mm}$. And the absorption loss of the solid fibre end would be $130 \times 0.005=0.65 \mathrm{~dB}$. Then the total absorption loss would be $3.6 \mathrm{~dB}$, still low enough for many applications.

\section{CONCLUSION}

In conclusion, we have proposed a simple technique to realize a low-loss $\mathrm{THz}$ fibre. The introduction of the sub- 
wavelength core can lower the loss of $\mathrm{THz}$ wave. Numerical simulations demonstrate that the transmission efficiency is high at two ends of the low-loss fibre. The proposed fibre provide a simple $\mathrm{THz}$ technique for short distance $(<1 \mathrm{~m})$ transmission.

\section{ACKNOWLEDGEMENTS}

This work is supported by National Basic Research Program of China (973 Program) (Grant No. 2011CB013004), the Priority Academic Program Development of Universities in Jiangsu Province, Universities Natural Science Research Project of Jiangsu Province (Grant No. 11KJA460003), and Photonic Manufacturing Science and Technology Key Laboratory Open Fund of Jiangsu Province (Grant No. GZ201114), and the Qing Lan Project of Jiangsu Province.

\section{References}

[1] P. H. Siegel, "Terahertz technology," IEEE Trans. Microw. Theory Tech. 50, 910-928 (2002).

[2] M. Tonouchi, "Cutting-edge terahertz technology," Nature Photon. 1, 97-105 (2007).

[3] P. Han, M. Tani, M. Usami, S. Kono, R. Kersting, and X. C. Zhang, "A direct comparison between terahertz time-domain spectroscopy and far-infrared Fourier transform spectroscopy," J. Appl. Phys. 89, 2357-2359 (2001).

[4] B. Clough, J. Liu, and X. C. Zhang, "All air-plasma terahertz spectroscopy," Opt. Lett. 36, 2399-2401 (2011).

[5] M. Seo, A. Adam, J. Kang, J. Lee, K. Ahn, Q. Park, P. Planken, and D. Kim, "Near field imaging of terahertz focusing onto rectangular apertures," Opt. Express 16, 20484-20489 (2008).

[6] V. P. Wallace, E. MacPherson, J. A. Zeitler, and C. Reid, "Threedimensional imaging of optically opaque materials using nonionizing terahertz radiation," J. Opt. Soc. Am. A 25, 3120-3133 (2008).
[7] R. Degl'Innocenti, M. Montinaro, J. Xu, V. Piazza, P. Pingue, A. Tredicucci, F. Beltram, H. Beere, and D. Ritchie, "Differential near-field scanning optical microscopy with $\mathrm{THz}$ quantum cascade laser sources," Opt. Express 17, 23785-23792 (2009).

[8] A. Hassani, A. Dupuis, and M. Skorobogatiy, "Porous polymer fibers for low-loss Terahertz guiding," Opt. Express 16, 6340-6351 (2008).

[9] D. Chen, and H. Chen, "A novel low-loss Terahertz waveguide: Polymer tube," Opt. Express 18, 3762-3767 (2010).

[10] K. Nielsen, H. K. Rasmussen, P. U. Jepsen, and 0. Bang, “Porouscore honeycomb bandgap THz fiber," Opt. Lett. 36, 666-668 (2011).

[11] A. Dupuis, K. Stoeffler, B. Ung, C. Dubois, and M. Skorobogatiy, "Transmission measurements of hollow-core THz Bragg fibers," J. Opt. Soc. Am. B 28, 896-907 (2011).

[12] S. Atakaramians, S. Afshar V, B. M. Fischer, D. Abbott, and T. M. Monro, "Porous fibers: a novel approach to low loss THz waveguides," Opt. Express 16, 8845-8854 (2008).

[13] A. Dupuis, A. Mazhorova, F. Désévédavy, M. Rozé, and M. Skorobogatiy, "Spectral characterization of porous dielectric subwavelength THz fibers fabricated using a microstructured molding technique," Opt. Express 18, 13813-13828 (2010).

[14] M. Rozé, B. Ung, A. Mazhorova, M. Walther, and M. Skorobogatiy, "Suspended core subwavelength fibers: towards practical designs for low-loss terahertz guidance," Opt. Express 19, 91279138 (2011).

[15] D. Chen, "Mode property of Terahertz polymer tube," J. Lightw. Technol. 28, 2708-2713 (2010).

[16] S. Selleri, L. Vincetti, A. Cucinotta, and M. Zoboli, “Complex FEM modal solver of optical waveguides with PML boundary conditions," Opt. Quantum Electron. 33, 359-371 (2001).

[17] W. Huang, and C. Xu, "Simulation of three-dimensional optical waveguides by a full-vector beam propagation method," IEEE J. Quantum Elect. 29, 2639-2649 (1993).

[18] G. R. Hadley, "Wide-angle beam propagation using Padé approximant operators," Opt. Lett. 17, 1426-1428 (1992). 